\title{
PROMETHEUS - THE DISTRIBUTED DIGITAL IMAGE ARCHIVE FOR RESEARCH AND EDUCATION GOES INTERNATIONAL!
}

\author{
Lisa Dieckmann \\ prometheus \\ c/o Kunsthistorisches Institut \\ University of Cologne \\ Albertus-Magnus-Platz \\ 50923 Cologne \\ Germany \\ tel: +49 221 470-4476, fax: *49 221 470-5044 \\ http://www.prometheus-bildarchiv.de
}

\begin{abstract}
Prometheus (http://www.prometheus-bildarchiv.de) is a distributed digital image archive for Art history, Archeology and Cultural Sciences. It connects image databases (47 as of May 2008), which differ in structure, volume and content, and renders these images (over 520.000 as of May 2008) accessible for research and education. A comfortable search interface for image-browsing and different sorting facilities make it easy to find and arrange individual images. Furthermore, two important co-operations are now facilitating the management of the reproduction rights. Thanks to recent technological improvement promethe us will from now on be an interesting research tool for users all over the world. Non-German speakers can fill in English terms when consulting the archive and get impressive results.
\end{abstract}

\section{INTRODUCTION}

In 2001 the idea of prometheus was born to fulfil an important need for Art History, Archeology and other Cultural Sciences that rely on the image as the main medium of discourse - a digital image archive for research and education.

The goal was not to stick to old slide-archive-traditions and to maintain databases at every single institute BUT to pool the resources of these institutions to make accessible as many good quality, high-resolution digital images as possible for research and education on a grand scale. Therefore the main aim was to connect various heterogeneous and topographically distributed digital image databases via internet similar to bibliographic network systems but with a different approach: individuality instead of standardisation. Furthermore, we intended to break down borders by integrating neighbouring disciplines to open perspectives for future research and education. We also wanted to provide didactic units to support academic teaching and learning with and via digital images.

With considerable funding from the German Federal Ministry of Education and Research from 2001 to 2004 pro me the u s could go about it.

Since 2002 prometheus has enabled a convenient search for images on a common user interface that joins various image archives and databases put up by university institutes, research facilities, museums and other institutions

As regards the total of images (over 520,000 high-resolution images as of March 2008) and connected databases (47), prometheus is now the largest distributed digital image archive in Europe and continuously increases in volume because of new 
image databases being integrated. Each image archive in prometheus brings in its own specific focus. The whole spectrum of the Arts and Cultural Sciences is on view in prometheus: from attic vases and ancient sculpture via gothic cathedrals and famous Italian masters to modern and contemporary art and new media. Historic postcards from the $19^{\text {th }}$ century and 2.000 videos amplify the archive's scope.

Having achieved the goals as they were defined at the outset and watching prometheus grow in scale and professional acceptance we found many more fields to turn to:

Because of the broad approval in German-speaking countries and responding to the rising interest voiced in other European countries, we provided for an English version of our website and integrated an English-German vocabulary to make bilingual usage possible.

Prometheus does not 'own' any single image. The individual databases are responsible for copyright issues concerning their images or the images they provide. However, prometheus has always demonstrated a keen interest in being informed on copyright legislation, in advising its partners on actions to take, and in finding solutions so that open access to cultural goods is guaranteed as a natural prerogative of research and education. Concerning the copyright on works of art and photographies, prometheus has contracted two types of cooperation: one with the collecting society (VG BildKunst, http://www.bildkunst.de/) and the other with a commercial image archive the 'Bildarchiv Preußischer Kulturbesitz' (bpk, http://www.bpk-images.de/) in Berlin. These arrangements provide models for future modes of copyright and publication management in order to facilitate the scientific use of images of fine art objects.

Before going into the details, let's first focus on the image archive and its structure itself, the web application and its features.

\section{IMAGE ARCHIVE}

\section{Technical data and structure}

The technical base of prometheus is the Web Application Framework Ruby on Rails with the search engine Ferret behind it.

In contrast to some earlier attempts (e.g. initiatives like HIDA/MIDAS), prometheu s does not try to impose a particular database upon all partners or provide particular standards. The only precondition to enter cooperation is that the database defines three basic elements (the work's title, the image itself, information on the copyright). XML is used as the only web-based standard that doesn't depend on any further specification.

Our central server holds profiles of the individual databases we work with. These profiles specify how the consultation of each single database has to be formulated and indexed and how the answer has to be processed in order to guarantee smooth adjustment.

\section{Web application}

After a revision of the software in March 2008, new methods are now implemented to facilitate work with the image archive and the built-in-tools.

The search interface offers you advanced search functions. You can use Boolean operators AND, NOT and OR as a way of specifying your research entry by indicating the relationships between your search terms. Furthermore you can use special query 
syntax like phrase search, wildcards, fuzzy search or boosting to modify your search query.

You may also want to restrict or specify the search pool by selecting individual databases or groups of databases. The databases are qualified by different criteria such as location, focus regarding content, function (institutional database, museum database and research database).

Regarding the search results you also have different possibilities. First of all you can sort your results according to criteria such as artist, title, location, credits or database, which facilitates the locating and grouping of relevant images. Additionally you may arrange your results individually. That means that you can choose between different amounts of records displayed or between two display modes: list or gallery (Figure 1).

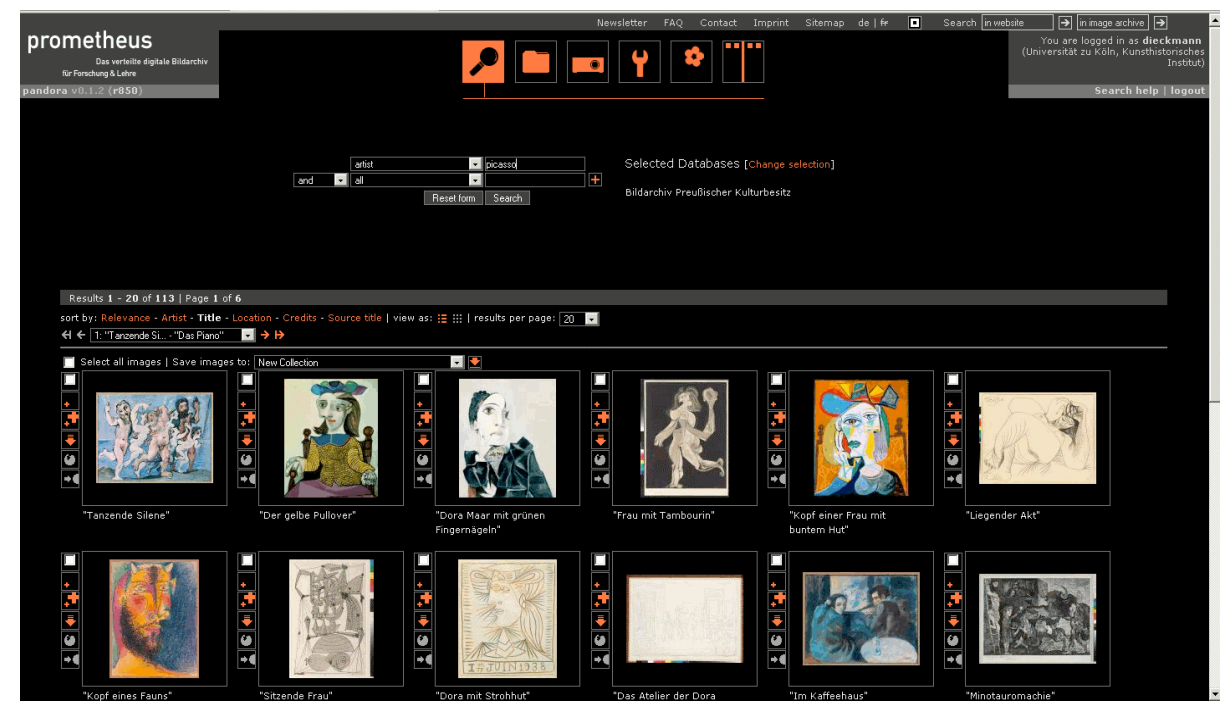

Figure 1. Result list with display mode 'gallery'

With a single click on the image you get the full view with a large size image and all the information that is provided by the respective database (Fig. 2).

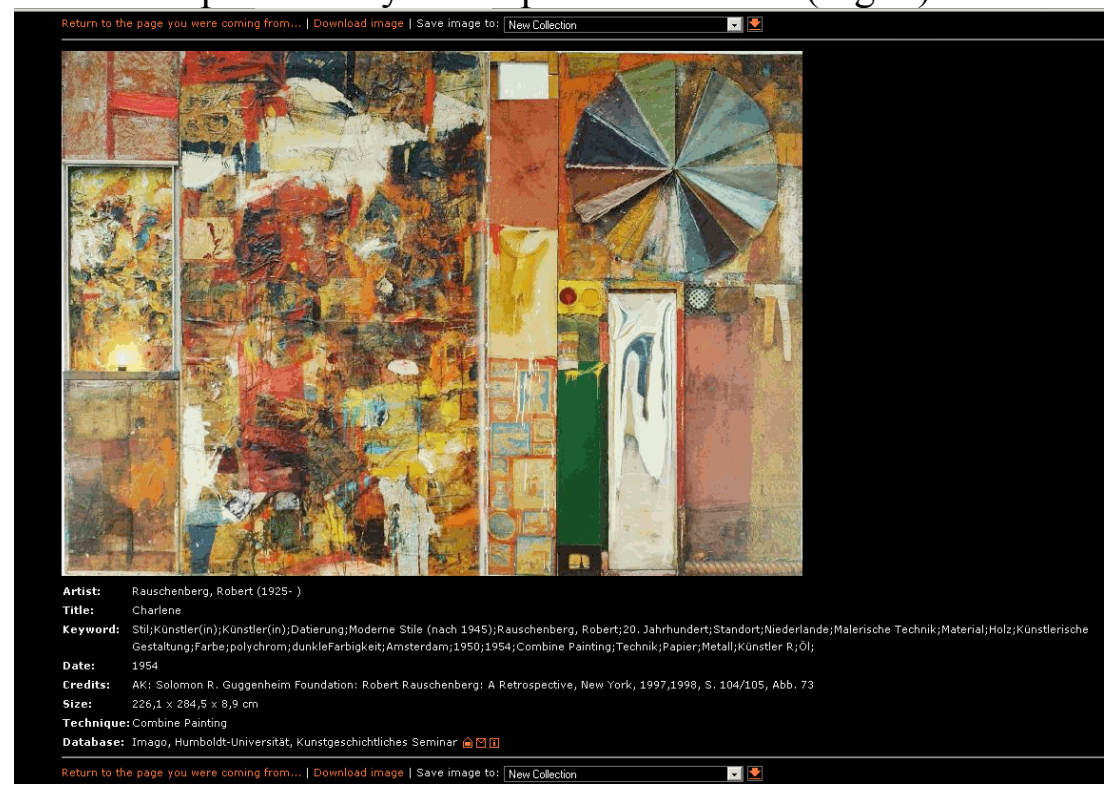

Figure 2. Full view with a large size image and further information 
In addition to the image retrieval prometheus offers the possibility to create personal collections (Fig. 3) from the search results list in order to compile images for a special topic (i.e. for a class or paper).

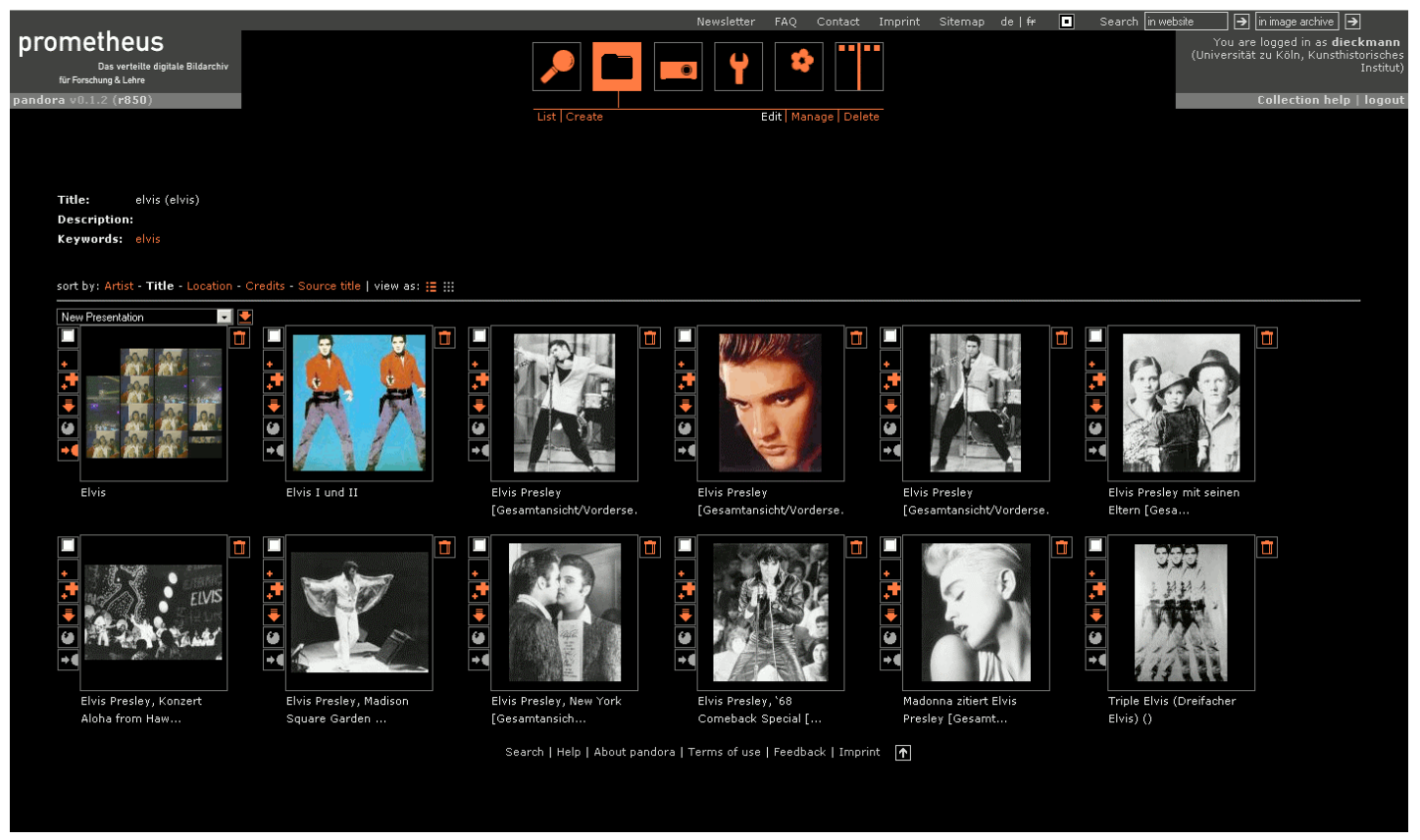

Figure 3. Collection

The collections can be linked with keywords and edited to become presentations (Fig. 4). The arrangement of the slots and the number of images in one slot is flexible. Your presentation's structure and optics remain flexible and basically up to you.

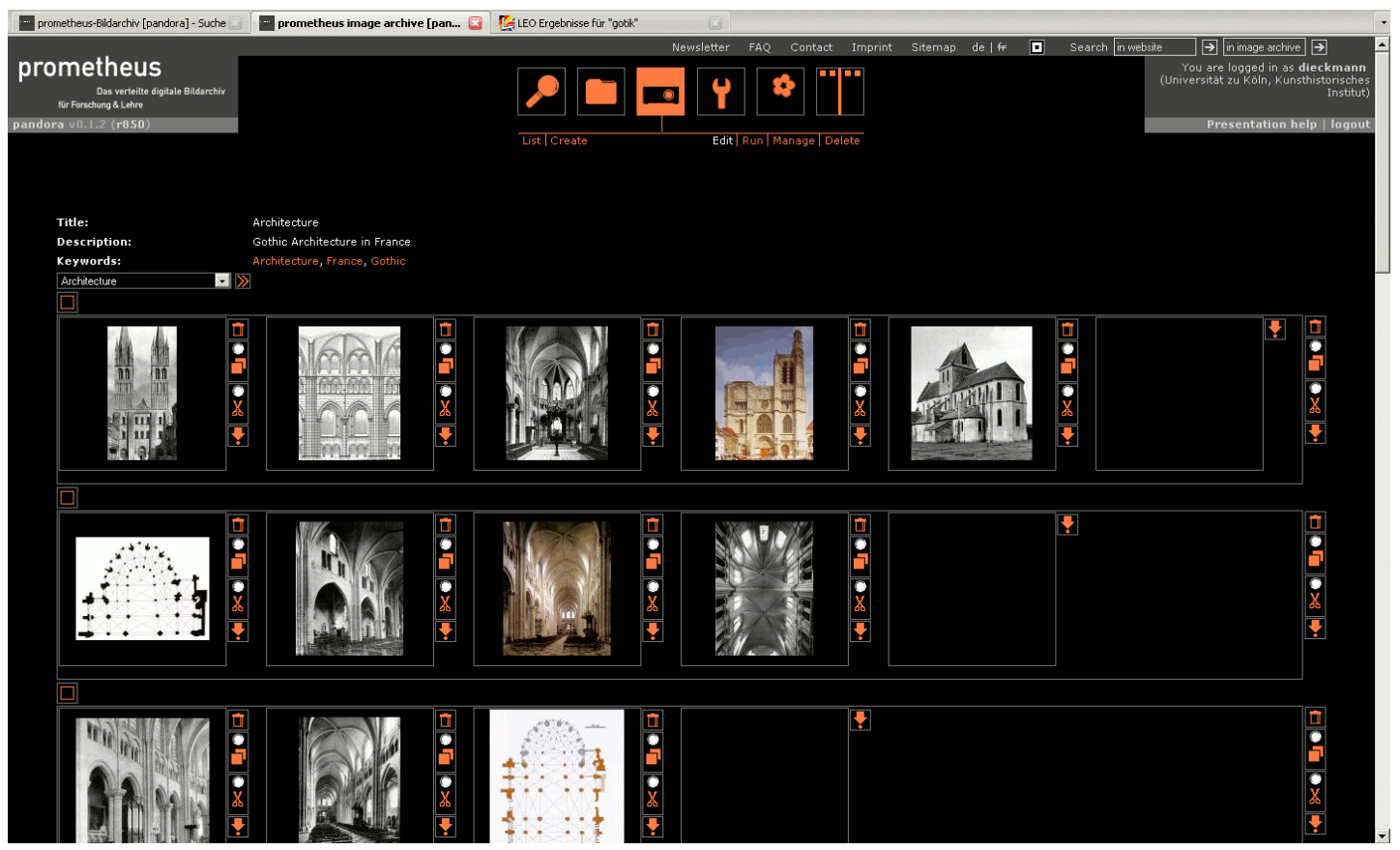

Figure 4. Presentation 
If you want to add local images to your presentation, we suggest a simple tool, free of charge, which is called "editor \& projector". It is very easy to manage and comes with some great features like the dynamic zoom.

prometheus provides also a variety of didactic units on the website to support academic teaching and learning and, moreover, besides the 'editor \& projector' further tools to use and handle digital images. Among them figures DILPS (Distributed Image Library Processing System, http://www.dilps.net/), a web-based open source software for managing and searching digital images. DILPS is one of the instruments we recommend to institutions that plan to digitize their images. The databases built with DILPS can be hosted by prometheus, if the institution has no capacities to maintain a server of their own.

\section{Bilingualism}

Intending to widen the group of users on a European scale we support bilingual searches and integrated an English-German vocabulary. In order to formulate queries in English and retrieve objects in German prometheus uses "lingo", an open source indexing system for research and education. Lingo is a joint development of Klaus Lepsky and John Vorhauer. (http://lex-lingo.blogspot.com/)

The main functions of lingo are identification of (i.e. reduction to) basic word form by means of a simple suffix list, algorithmic decomposition, dictionary-based lexical synonymisation and identification of phrases, and generic identification of phrases or word sequences based on succession of word classes. Lingo knows two different kinds of dictionaries: dictionaries, which simply consist of word forms, basic word form of a word and the appropriate word class; and dictionaries, which contain synonyms without any morpho-syntactic information. These dictionaries include e.g. the English translation of German words. The structure of the lingo dictionaries is as simple as possible. It is very easy and comfortable to create, to insert or maintain your own dictionaries that can be used to describe your collection.

\section{CONTINUITY AND FINANCING}

Since 2004 the non-profit association ,prometheus - the distributed digital image archive for research and education e.V." has guaranteed the long-term existence and further development of the former project. The head office is situated in Cologne, at the institute of art history at the University of Cologne.

Our financing relies on a combination of third-party-funds and license fees. The license fees are a present rather low compared to the ones demanded by similar archives, and we consider a moderate upgrade in 2009.

The licence fees are usually covered by the university libraries - as is the case with digital dictionaries and other research tools that are used by a broad range of academic disciplines. Campus and institutional fees enable free access for a considerably large group of users, among them students and learners of all kinds.

promethe us cooperates with the centre of university librairies in Cologne which manages the marketing of licences for us.

\section{COPYRIGHT AND PUBLICATION MANAGEMENT}

Use of Images for Research and Education 
The images and the media files (e.g. videos) available in promethe us may be used as instruction materials by academic institutions and schools as well as for individual scientific purposes without paying a fee or obtaining a license. Usage other than for research and education requires the consideration of possible third-party copyrights, and might necessitate obtaining the consent of the copyright holder.

\section{Copyright}

\section{Work of art}

The copyright holder of a work of art is the artist. Utilization rights remain the property of the artist, and can be transferred to his/her heirs for up to 70 years after the demise of the artist (UrhG §64).

After that time, the copyright expires, and the work of art enters the public domain. This definition makes clear that a copyright exists only, when a specific work of art was created by an artist who died less than 70 years ago.Utilization rights of all artists for the years before the $70^{\text {th }}$ anniversary of their demise are managed by the so called collecting society (VG Bild-Kunst) in Bonn, Germany.

promethe u s has concluded an agreement with the 'VG Bild-Kunst'. According to this agreement it is permitted that the images in question are made available through the various image databases cooperating in prometheus It is also allowed to use these images world wide for non-commercial research and educational purposes.

\section{Reproduction of the work of art}

Photographic reproductions of works of art are protected by ancillary copyright as well (UrhG§72, Right to Use). Photos/slides are products of a straightforward photographic process or a process analogous to photography.

Copyright protection terminates 50 years after the creation of the photograph/slide, or 50 years after its first authorized publication. If a more artistic photograph has been produced, for example by choosing a certain angle or special lighting, it is defined as a photographic work of art, and is protected by copyright for up to 70 years after the photographer's death. As a rule, photos taken of three-dimensional originals (sculptures, etc.) result in a photographic work of art.

Permission to use them requires authorization directly from the photographer or institution (e.g. image database, publisher). prometheus endeavours to name the respective copyright holder of the photography in question. Sometimes the user has to do the research concerning the copyright him- or herself. The source of the image that is part of the results data should be helpful here.

prometheus is interested in negotiating contracts with the copyright holders of photographies and photographic works of art, with photographers, image database holders, publishers etc., in order to enable us to provide or mediate permission for publication in non-profit, scientific publications.

We were able to negotiate an exemplary agreement of cooperation with the commercial image archive 'Bildarchiv Preußischer Kulturbesitz' (bpk) in Berlin. It permits the use of all the digital images that have been made available to prometheus by the bpk, free of charge in non-profit, scientific publications with a circulation of less than 1000 copies. The bpk, the museum and the photographer named in the picture credits have to be specified in the annotations to the published image. 
Without further request, a sample copy is to be sent to the museums the works of art come from. Obtaining legal approval of the copyright holder of the respective images is easily accomplished by using the publication function, provided by prometheus (Figure 5).

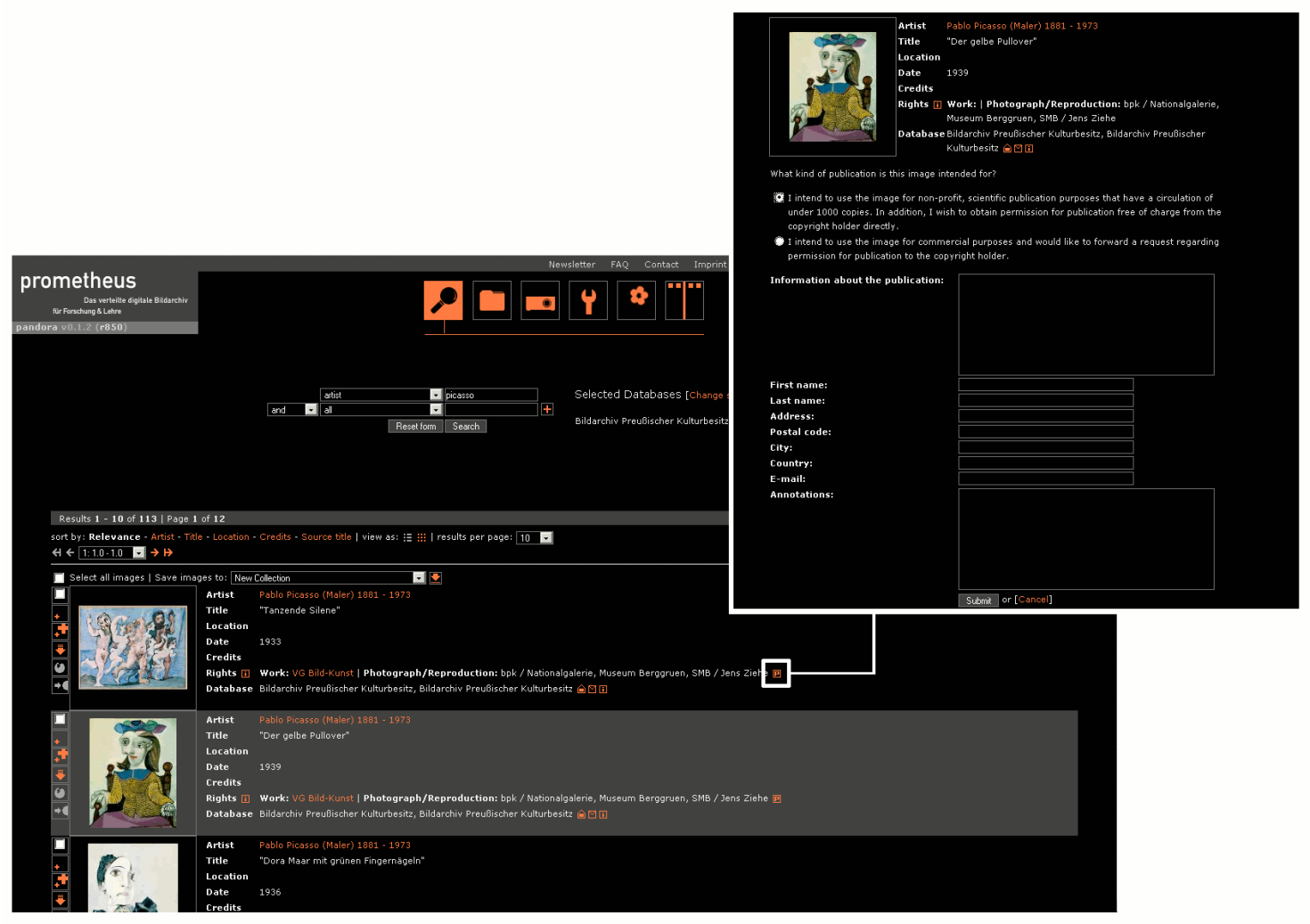

Figure 5. Result list with publication button and publication form 